\title{
NONLOCAL PdES ON GRAPHS FOR ACTIVE CONTOURS MODELS WITH APPLICATIONS TO IMAGE SEGMENTATION AND DATA CLUSTERING
}

O. Lézoray, A. Elmoataz

\author{
Université de Caen Basse-Normandie \\ GREYC UMR CNRS 6072, Caen, France
}

\begin{abstract}
We propose a transcription on graphs of recent continuous global active contours proposed for image segmentation to address the problem of binary partitioning of data represented by graphs. To do so, using the framework of Partial difference Equations (PdEs), we propose a family of nonlocal regularization functionals that verify the co-area formula on graphs. The gradients of a sub-graph are introduced and their properties studied. Relations, for the case of a sub-graph, between the introduced nonlocal regularization functionals and nonlocal discrete perimeters are exhibited and the co-area formula on graphs is introduced. Finally, nonlocal global minimizers can be considered on graphs with the associated energies. Experiments show the benefits of the approach for nonlocal image segmentation and high dimensional data clustering.
\end{abstract}

Index Terms - Partial difference Equations (PdEs), Graphs, Global active contours, Nonlocal, Clustering.

\section{INTRODUCTION}

With the advent of our digital world, much different kind of data are now available. On the contrary to classical images and videos, these data do not necessarily lie on a Cartesian grid and can be irregularly distributed. To represent a large number of data domains (images, meshes, social networks, etc.), the most natural and flexible representation consists in using weighted graphs by modeling neighborhood relationships. Therefore, there is much interest in the transposition of signal and image processing tools for the processing of functions on graphs, e.g., spectral graph wavelets [1] or PDE on graphs $[2,3]$. In this paper, we consider active contour models. They can be casted in two models: geometric models with level sets and variational models with Total Variation. We consider the second model and extend it to the processing of arbitrary data on graphs as well as unify their local and nonlocal processing for images. To do this transcription, we use the framework of PdEs [3] along with a family of discrete gradients. We introduce nonlocal perimeters as well as the coarea on graphs. We then consider nonlocal global minimizers for segmentation and clustering and provide an application example with nonlocal Chan-Vese on graphs.

\author{
V.-T. Ta
}

\author{
LaBRI (Université de Bordeaux, CNRS, IPB) \\ Talence, France
}

\section{PARTIAL DIFFERENCE EQUATIONS ON GRAPHS}

In this section, we recall definitions and operators on graphs. All the operators presented in this section are naturally local and nonlocal [3]. Indeed, local and nonlocal configurations are expressed by graph topology and edges' weights.

\subsection{Preliminary notations}

A weighted graph $\mathcal{G}=(\mathcal{V}, \mathcal{E}, w)$ consists in a finite set $\mathcal{V}$ of vertices and a finite set $\mathcal{E} \subset \mathcal{V} \times \mathcal{V}$ of weighted edges. We assume $\mathcal{G}$ to be undirected, with no self-loops and no multiple edges. Let $e_{i j}=\left(v_{i}, v_{j}\right)$ be the edge of $\varepsilon$ that connects vertices $v_{i}$ and $v_{j}$ of $\mathcal{V}$. Its weight, denoted by $w_{i j}=w\left(v_{i}, v_{j}\right)$, represents the similarity between its vertices and is computed using a positive symmetric function $w$. Notation $v_{i} \sim v_{j}$ is also used to denote two adjacent vertices. The volume of a set of vertices $\mathcal{A}$ can be computed by $\operatorname{vol}(\mathcal{A})=\sum_{v_{i} \in \mathcal{A}} d_{\mathcal{A}}\left(v_{i}\right)$ with $d_{\mathcal{A}}\left(v_{i}\right)=\sum_{v_{j} \sim v_{i}} w\left(v_{i}, v_{j}\right), \forall v_{i} \in \mathcal{A}$ the degree of a vertex relatively to a set of vertices $\mathcal{A}$. Let $\mathcal{H}(\mathcal{V})$ be the Hilbert space of real-valued functions defined on the vertices of a graph. A function $f: \mathcal{V} \rightarrow \mathbb{R}$ of $\mathcal{H}(\mathcal{V})$ assigns a real value $x_{i}=f\left(v_{i}\right)$ to each vertex $v_{i} \in \mathcal{V}$. By analogy with functional analysis on continuous spaces, the integral of a function $f \in \mathcal{H}(\mathcal{V})$, over the set of vertices $\mathcal{V}$, is defined as $\int_{\mathcal{V}} f=\sum_{\mathcal{V}} f$. Similarly, let $\mathcal{H}(\mathcal{E})$ be the space of real-valued functions defined on the edges of $\mathcal{G}$. Both Hilbert spaces are endowed with the usual inner products.

\subsection{Difference operators}

Let $f: \mathcal{V} \rightarrow \mathbb{R}$ be a function of $\mathcal{H}(\mathcal{V})$. The difference operator [3] of $f$, noted $d_{w}: \mathcal{H}(\mathcal{V}) \rightarrow \mathcal{H}(\mathcal{E})$, is defined on an edge $\left(v_{i}, v_{j}\right) \in \mathcal{E}$ by: $\left(d_{w} f\right)\left(v_{i}, v_{j}\right)=w\left(v_{i}, v_{j}\right)^{1 / 2}\left(f\left(v_{j}\right)-f\left(v_{i}\right)\right)$. The directional derivative of $f$, at a vertex $v_{i} \in \mathcal{V}$, along an edge $e_{i j}=\left(v_{i}, v_{j}\right)$, is defined as $\left.\frac{\partial f}{\partial e_{i j}}\right|_{v_{i}}=\partial_{v_{j}} f\left(v_{i}\right)=$ $\left(d_{w} f\right)\left(v_{i}, v_{j}\right)$. Based on the difference operator, the weighted morphological external and internal difference operators are respectively [4]: $\left(d_{w}^{+} f\right)\left(v_{i}, v_{j}\right)=\max \left(0,\left(d_{w} f\right)\left(v_{i}, v_{j}\right)\right)$ and $\left(d_{w}^{-} f\right)\left(v_{i}, v_{j}\right)=-\min \left(0,\left(d_{w} f\right)\left(v_{i}, v_{j}\right)\right)$. The correspond- 
ing external and internal partial derivatives are $\partial_{v}^{+} f\left(v_{i}\right)=$ $\left(d_{w}^{+} f\right)\left(v_{i}, v_{j}\right)$ and $\partial_{v}^{-} f\left(v_{i}\right)=\left(d_{w}^{-} f\right)\left(v_{i}, v_{j}\right)$. The adjoint of the difference operator, noted $d_{w}^{*}: \mathcal{H}(\mathcal{E}) \rightarrow \mathcal{H}(\mathcal{V})$, is a linear operator defined, for all $f \in \mathcal{H}(\mathcal{V})$ and all $H \in \mathcal{H}(\mathcal{E})$, by $\left\langle d_{w} f, H\right\rangle_{\mathcal{H}(\mathcal{E})}=\left\langle f, d_{w}^{*} H\right\rangle_{\mathcal{H}(\mathcal{V})}$. This adjoint operator $d_{w}^{*}$, of a function $H \in \mathcal{H}(\mathcal{E})$, can by expressed at a vertex $v_{i} \in \mathcal{V}$ by the following expression: $\left(d_{w}^{*} H\right)\left(v_{i}\right)=-d i v_{w}(H)\left(v_{i}\right)=$ $\sum_{v_{j} \sim v_{i}} w\left(v_{i}, v_{j}\right)^{1 / 2}\left(H\left(v_{j}, v_{i}\right)-H\left(v_{i}, v_{j}\right)\right)$.

\subsection{Gradients and norms}

The weighted gradient operator of a function $f \in \mathcal{H}(\mathcal{V})$, at a vertex $v_{i} \in \mathcal{V}$, is the vector operator defined by $\left(\nabla_{\mathbf{w}} \mathbf{f}\right)\left(\mathbf{v}_{\mathbf{i}}\right)=$ $\left[\partial_{v_{j}} f\left(v_{i}\right): v_{j} \sim v_{i}\right]^{T}$. The $\mathcal{L}_{p}$ norm of this vector is defined by [3]: $\quad\left\|\left(\nabla_{\mathbf{w}} \mathbf{f}\right)\left(\mathbf{v}_{\mathbf{i}}\right)\right\|_{p}^{p}=\sum_{v_{j} \sim v_{i}} w\left(v_{i}, v_{j}\right)^{p / 2}\left|f\left(v_{j}\right)-f\left(v_{i}\right)\right|^{p}$. Similarly, we have $\left(\nabla_{\mathbf{w}}^{ \pm} \mathbf{f}\right)\left(\mathbf{v}_{\mathbf{i}}\right)=\left[\partial_{v_{j}}^{ \pm} f\left(v_{i}\right): v_{j} \sim v_{i}\right]^{T}$ and $\left\|\left(\nabla_{\mathbf{w}}^{ \pm} \mathbf{f}\right)\left(\mathbf{v}_{\mathbf{i}}\right)\right\|_{p}^{p}=\sum_{v_{j} \sim v_{i}} w\left(v_{i}, v_{j}\right)^{p / 2}\left|M^{ \pm}\left(0, f\left(v_{j}\right)-f\left(v_{i}\right)\right)\right|^{p}$ with $M^{+}=\max$ and $M^{-}=\min$. These norms exhibit the following property: $\left\|\left(\nabla_{\mathbf{w}} \mathbf{f}\right)\left(\mathbf{v}_{\mathbf{i}}\right)\right\|_{p}^{p}=\left\|\left(\nabla_{\mathbf{w}}^{+} \mathbf{f}\right)\left(\mathbf{v}_{\mathbf{i}}\right)\right\|_{p}^{p}+$ $\left\|\left(\nabla_{\mathbf{w}}^{-} \mathbf{f}\right)\left(\mathbf{v}_{\mathbf{i}}\right)\right\|_{p}^{p}$.

\subsection{Anisotropic $p$-Laplace operator}

The weighted p-Laplace anisotropic operator of a function $f \in \mathcal{H}(\mathcal{V})$, noted $\Delta_{w, p}^{a}: \mathcal{H}(\mathcal{V}) \rightarrow \mathcal{H}(\mathcal{V})$, is defined by: $\left(\Delta_{w, p}^{a} f\right)\left(v_{i}\right)=\frac{1}{2} d_{w}^{*}\left(\left|\left(d_{w} f\right)\left(v_{i}, v_{j}\right)\right|^{p-2}\left(d_{w} f\right)\left(v_{i}, v_{j}\right)\right)$. Using the definitions of $d_{w}$ and $d_{w}^{*}$, we obtain this expression [5]: $\left(\Delta_{w, p}^{a} f\right)\left(v_{i}\right)=\sum_{v_{j} \sim v_{i}}\left(\gamma_{w, p}^{a} f\right)\left(v_{i}, v_{j}\right)\left(f\left(v_{i}\right)-f\left(v_{j}\right)\right)$. with $\left(\gamma_{w, p}^{a} f\right)\left(v_{i}, v_{j}\right)=w_{i j}^{p / 2}\left|f\left(v_{i}\right)-f\left(v_{j}\right)\right|^{p-2}$. This operator is nonlinear if $p \neq 2$. In this latter case, it corresponds to the combinatorial graph Laplacian.

\section{VARIATIONAL FRAMEWORK OF ACTIVE CONTOURS ON GRAPHS}

In this section, we introduce a family of nonlocal regularization functionals that verify the co-area formula for $p=1$. The gradients of a sub-graph are introduced and their properties studied. Then, we show that there are strong relations, for the case of a sub-graph, between the introduced nonlocal regularization functionals and nonlocal discrete perimeters. Finally nonlocal global minimizers can be considered on arbitrary graphs with the associated nonlocal energies.

\subsection{Nonlocal regularization functionals}

Let us consider nonlocal regularization functionals based on weighted total variation $\left(T V_{w}\right)$ on graphs $R_{w, p}: \mathcal{H}(V) \rightarrow \mathbb{R}$ of a function $f \in \mathcal{H}(V): R_{w, p}(f)=\sum_{v_{i} \in \mathcal{V}}\left\|\mathbf{D}_{\mathbf{w}} \mathbf{f}\left(\mathbf{v}_{\mathbf{i}}\right)\right\|_{p}^{p}$ with $0<p<+\infty$. $\quad \mathbf{D}_{\mathrm{w}}$ is a discrete gradient operator that can be among $\nabla_{\mathbf{w}}$ and $\nabla_{\mathbf{w}}^{ \pm}$. By replacing $\mathbf{D}_{\mathbf{w}}$ with the previous previous discrete gradients one obtains several functionals: $R_{w, p}(f)=\sum_{v_{i} \in \mathcal{V}} \sum_{v_{j} \sim v_{i}} w\left(v_{i}, v_{j}\right)^{p / 2}\left|f\left(v_{j}\right)-f\left(v_{i}\right)\right|^{p}$, and
$R_{w, p}^{ \pm}(f)=\sum_{v_{j} \in \mathcal{V}} \sum_{v_{j} \sim v_{i}} w\left(v_{i}, v_{j}\right)^{p / 2}\left|M^{ \pm}\left(0, f\left(v_{j}\right)-f\left(v_{i}\right)\right)\right|^{p}$ with property $R_{w, p}(f)=R_{w, p}^{+}(f)+R_{w, p}^{-}(f)$. Finally, using the chain rule, we can show [5] that $\frac{\partial R_{w, p}^{a}(f)}{\partial f\left(v_{i}\right)}=2 p\left(\Delta_{w, p}^{a} f\right)\left(v_{i}\right)$.

\subsection{Nonlocal perimeters and co-area on graphs}

\subsubsection{Gradients of a sub-graph}

Let $\mathcal{A}$ be a set of connected vertices with $\mathcal{A} \subset \mathcal{V}$. We denote by $\partial^{+} \mathcal{A}$ and $\partial^{-} \mathcal{A}$, the external and internal boundary sets of $\mathcal{A}$, respectively. $\mathcal{A}^{-}=\mathcal{V} \backslash \mathcal{A}$ is the complement of $\mathcal{A}$. For a given vertex $v_{i} \in \mathcal{V}, \partial^{+} \mathcal{A}=\left\{v_{i} \in \mathcal{A}^{-}: \exists v_{j} \in \mathcal{A}\right.$ with $\left.\left(v_{i}, v_{j}\right) \in \mathcal{E}\right\}$ and $\partial^{-} \mathcal{A}=\left\{v_{i} \in \mathcal{A}: \exists v_{j} \in \mathcal{A}^{-}\right.$with $\left.\left(v_{i}, v_{j}\right) \in \mathcal{E}\right\}$. We now consider the gradients of a sub-graph and study their properties. We denote by $\chi_{\mathcal{A}}$ the indicator function defined by $\chi: \mathcal{V} \rightarrow\{0,1\}$.

\section{Proposition 1}

For a given vertex, if $v_{i} \in \mathcal{A}$, then $\chi_{\mathcal{A}}\left(v_{i}\right)=1$ and $\chi_{\mathcal{A}}\left(v_{i}\right)=0$ otherwise. The $\mathcal{L}_{p}$-norm of the directional gradient of the indicator function is defined as:

$$
\left\|\nabla_{\mathbf{w}}^{ \pm} \chi_{\mathcal{A}}\left(\mathbf{v}_{\mathbf{i}}\right)\right\|_{p}^{p}=\chi_{\partial^{ \pm} A}\left(v_{i}\right) \sum_{v_{j} \sim v_{i}, v_{j} \in \mathcal{A}^{ \pm}} w\left(v_{i}, v_{j}\right)^{p / 2}
$$

with the notation $\mathcal{A}^{+}=\mathcal{A}$ and $\mathcal{A}^{-}=\mathcal{V} \backslash \mathcal{A}$.

Proof. By studying cases where $v_{i} \in \mathcal{A}, v_{i} \notin \mathcal{A}$ and similarly with the neighbors of $v_{i}$, we can prove that only the inner and outer set of vertices $\partial^{ \pm} A$ are concerned.

This leads to the following property:

$$
\left\|\nabla_{\mathbf{w}} \chi_{\mathcal{A}}\left(\mathbf{v}_{\mathbf{i}}\right)\right\|_{p}^{p}=\left\|\nabla_{\mathbf{w}}^{+} \chi_{\mathcal{A}}\left(\mathbf{v}_{\mathbf{i}}\right)\right\|_{p}^{p}+\left\|\nabla_{\mathbf{w}}^{-} \chi_{\mathcal{A}}\left(\mathbf{v}_{\mathbf{i}}\right)\right\|_{p}^{p}
$$

\subsubsection{Nonlocal functionals and nonlocal perimeters}

Now, we show that there are strong relations, for the case of a sub-graph, between the previous discrete regularization functionals and discrete perimeters.

\section{Proposition 2}

By replacing $f$ by the indicator function $\chi_{\mathcal{A}}$ in the regularization functionals, one has: $R_{w, p}^{ \pm}\left(\chi_{\mathcal{A}}\right)=\frac{1}{2} \operatorname{vol}(\partial A)$.

Proof. Using equation (1) and the nonlocal functionals, $\quad R_{w, p}^{+}\left(\chi_{\mathcal{A}}\right)=\sum_{v_{i} \in \mathcal{V}} \sum_{v_{j} \sim v_{i}, v_{j} \in \mathcal{A}} w\left(v_{i}, v_{j}\right)^{p / 2} \chi_{\partial^{+} A}\left(v_{i}\right)=$ $\sum_{v_{i} \in \partial^{+} A} \sum_{v_{j} \in \partial^{-} A} w\left(v_{i}, v_{j}\right)^{p / 2}=\sum_{v_{i} \in \partial^{+} A} d_{\partial^{-} A}\left(v_{i}\right)=\frac{1}{2} \operatorname{vol}(\partial A)$. Similarly, one has $R_{w, p}^{-}\left(\chi_{\mathcal{A}}\right)=\frac{1}{2} \operatorname{vol}(\partial A)$, and $R_{w, p}\left(\chi_{\mathcal{A}}\right)=$ $\operatorname{vol}(\partial \mathcal{A})$.

All the previous functionals can be then seen as the inner $\left(R_{w, p}^{-}\left(\chi_{A}\right)\right)$, outer $\left(R_{w, p}^{+}\left(\chi_{A}\right)\right)$ or global $\left(R_{w, p}\left(\chi_{A}\right)\right) p$-cost of a cut between two set of vertices $\mathcal{A}$ and $\mathcal{A}^{-}$. As a consequence, all these previous expressions can be seen as nonlocal discrete perimeters of a sub-graph:

$$
2 \operatorname{Per}_{w, p}^{ \pm}(\mathcal{A})=\operatorname{Per}_{w, p}(\mathcal{A})=2 R_{w, p}^{ \pm}\left(\chi_{A}\right)=R_{w, p}\left(\chi_{\mathcal{A}}\right)
$$


This shows explicitly the relations between the functionals we defined with Total Variation and nonlocal discrete perimeters of a sub-graph. Interestingly, we can recover on grid graphs with specific weights, a discrete analogue of the continuous nonlocal perimeters introduced in [6].

\subsubsection{Co-area formula on weighted graphs}

Now we show that for $p=1$, the introduced nonlocal functionals do verify the co-area formula.

\section{Proposition 3}

For any function $f \in \mathcal{H}(\mathcal{V})$ and for $p=1$, one has the following property:

$$
R_{w, 1}^{*}(f)=\int_{-\infty}^{+\infty} R_{w, 1}^{*}\left(\chi_{\{f>t\}}\right) d t
$$

where $R_{w, 1}^{*}(f)$ is among $R_{w, 1}(f)$ and $R_{w, 1}^{ \pm}(f)$ with $\chi_{\{f>t\}}\left(v_{i}\right)$ $=0$ if $f\left(v_{i}\right)<t$ and $\chi_{\{f>t\}}\left(v_{i}\right)=1$ if $f\left(v_{i}\right) \geq t$.

Proof. All the functionals $R_{w, 1}^{*}$ appearing in (2) are of the form $R_{w, 1}(f)=\sum_{v_{i} \in \mathcal{V}} \sum_{v_{j} \sim v_{i}} w\left(v_{i}, v_{j}\right)\left|f\left(v_{j}\right)-f\left(v_{i}\right)\right|$ or $R_{w, 1}^{ \pm}(f)=\sum_{v_{i} \in \mathcal{V}} \sum_{v_{j} \sim v_{i}} w\left(v_{i}, v_{j}\right)\left(f\left(v_{j}\right)-f\left(v_{i}\right)\right)^{ \pm}$with $x^{+}=\max (0, x)$ is the nonnegative part of the real number $x\left(x^{-}=(-x)^{+}\right)$. Since $|a-b|=\int_{-\infty}^{+\infty}\left|\chi_{\{a>t\}}-\chi_{\{b>t\}}\right| d t$ and $(a-b)^{ \pm}=\int_{-\infty}^{+\infty}\left(\chi_{\{a>t\}}-\chi_{\{b>t\}}\right)^{ \pm} d t, R_{w, 1}^{*}$ clearly satisfies (2).

This result is different from [7] that assumes that the set of vertices of the graph belong to a submanifold and then make use of the co-area formula on manifolds. Our proposal does not need this assumption and is much simpler since have the co-area formula for any weighted graph with $p=1$.

\subsection{Nonlocal active contours models as global minimiz- ers for segmentation and clustering}

The usual drawback of active contours methods is the existence of local minimizers with the solution highly dependent of the initial condition. A recent method, introduced by Bresson and Chan $[8,7]$, proposes to redefine the active contour model into a model which gives global minimizers. Using our framework we show how we can introduce nonlocal global minimizers of active contours models on graphs. Let us consider this very general minimization problem on graphs:

$$
\min _{f: \mathcal{V} \rightarrow\{0,1\}}\left\{T V_{w}(f)+\lambda H\left(f, f^{0}\right)\right\}
$$

where $T V_{w}(f)$ is a weighted total variation of $f$. Both $T V_{w}(f)$ and $H\left(f, f^{0}\right)$ are considered as satisfying the co-area formula. When $\lambda \neq 0$, this energy can be considered as the nonlocal discrete analogue on graphs of [7]. When $\lambda=0$, it is the discrete analogue to the continuous min-cut of [9] since this reduces to minimize $\operatorname{Per}_{w, p}^{*}(\mathcal{A})=\operatorname{vol}(\partial \mathcal{A})=\operatorname{cut}\left(\mathcal{A}, \mathcal{A}^{-}\right)$. Now, we show how such a minimization can be solved but we restrict ourselves to the following minimization problem:

$$
\min _{\bar{f}: \mathcal{V} \rightarrow\{0,1\}}\left\{E_{w, 1}(f)=R_{w, 1}^{*}(f)+\lambda \sum_{v_{i} \in \mathcal{V}} r\left(f^{0}\left(v_{i}\right)\right) f\left(v_{i}\right)\right\}
$$

where $R_{w, 1}^{*}(f)$ is among $R_{w, 1}(f)$ and $R_{w, 1}^{ \pm}(f)$. As previously shown, the first part $R_{w, 1}^{*}(f)$ of the energy $E_{w, p}(f)$ satisfies the co-area formula. It can also be easily shown that it is also the case for the second part of the energy. Minimization problem (4) is non-convex and, as shown in [10] for the continuous analogue, can be reformulated to consider a convex relaxation problem to determine a global minimizing solution. Therefore, a new minimization problem is considered:

$$
\min _{\hat{f}: \mathcal{V} \rightarrow[0,1]}\left\{\hat{E}_{w, 1}(f)=R_{w, 1}^{*}(f)+\lambda \sum_{v_{i} \in \mathcal{V}} r\left(f^{0}\left(v_{i}\right)\right) f\left(v_{i}\right)\right\}
$$

Following the approach in [10], one can show, that every level set of a minimizer of (5) is a solution of the original optimization problem (4). As a consequence, to obtain a global solution $\bar{f}: \mathcal{V} \rightarrow\{0,1\}$ to the problem (4), one thresholds any function $\hat{f}: \mathcal{V} \rightarrow[0,1]$ that is a solution of $(5)$ and $\bar{f}=\chi_{A}(\hat{f})$. To establish the solution of the minimization problem (4), we can solve the following system of equations:

$$
\frac{\partial \hat{E}_{w, 1}(f)}{\partial f\left(v_{i}\right)}=\frac{\partial R_{w, 1}^{*}(f)}{\partial f\left(v_{i}\right)}+\lambda r\left(f^{0}\left(v_{i}\right)\right)=0, \quad \forall v_{i} \in \mathcal{V},
$$

First it is easy to show that one has the following relation $\frac{\partial R_{w, 1}(f)}{\partial f\left(v_{i}\right)}=2 \frac{\partial R_{w, 1}^{+}(f)}{\partial f\left(v_{i}\right)}=2 \frac{\partial R_{w, 1}^{-}(f)}{\partial f\left(v_{i}\right)}=2 \Delta_{w, 1}^{a} f\left(v_{i}\right)$. To approximate the solution of the minimization problem (5), the algorithms exposed in [7] can be used (dual/projection, graph cuts). We choose to use, for the sake of simplicity, the linearized GaussJacobi method and obtain the following iterative algorithm, $\forall v_{i} \in V$ :

$$
f^{(t+1)}\left(v_{i}\right)=\frac{-\lambda r\left(f^{0}\left(v_{i}\right)\right)+\alpha \sum_{v_{j} \sim v_{i}} \gamma_{w}^{f^{(t)}}\left(v_{i}, v_{j}\right) f^{(t)}\left(v_{j}\right)}{\alpha \sum_{v_{j} \sim v_{i}} \gamma_{w}^{f^{(t)}}\left(v_{i}, v_{j}\right)}
$$

where $\gamma_{w}^{f^{(t)}}\left(v_{i}, v_{j}\right)=w\left(v_{i}, v_{j}\right)^{1 / 2}\left|f^{t}\left(v_{i}\right)-f^{t}\left(v_{j}\right)\right|_{\varepsilon}^{-1}$. With $\alpha=$ 1 for $R_{w, 1}^{ \pm}(f)$ and $\alpha=2$ for $R_{w, 1}(f)$, and the initial condition $f^{(0)}=f^{0}$. The problem being relaxed on $\mathbb{R}$, at each iteration $t$, the solution is projected on $[0,1]$ by $\max \left(\min \left(f^{t}, 1\right), 0\right)$.

\section{APPLICATIONS}

In the following, we discuss the application of our proposed approach to Chan-Vese (CV) model. Since graphs of the arbitrary topologies can be considered, our approach enables to consider any type of discrete data that can be represented by a 
graph. Moreover, it can be used to segment image with nonlocal configurations and to cluster high dimensional data. We can directly express the discrete analogue on graphs of the $\mathrm{CV}$ model as the following minimization, $\forall v_{i} \in V$ :

$$
\min _{f: \mathcal{V} \rightarrow\{0,1\}}\left\{R_{w, 1}^{*}(f)+\lambda \sum_{v_{i} \in \mathcal{V}} f\left(v_{i}\right) m\left(c_{1}, v_{i}\right)+\left(1-f\left(v_{i}\right)\right) m\left(c_{2}, v_{i}\right)\right\}
$$

where $m\left(a, v_{i}\right)=\left(a-f^{0}\left(v_{i}\right)\right)^{2} . \quad c_{1}$ and $c_{2}$ are the means inside and outside the object. For a fixed $f$, the minimizer $\left(c_{1}(f), c_{2}(f)\right)$ can be explicitly computed. For fixed $\left(c_{1}, c_{2}\right)$, the minimization over $f$ can be solved with algorithm (7) with $r\left(f^{0}\left(v_{i}\right)\right)=\left(c_{1}-f^{0}\left(v_{i}\right)\right)^{2}-\left(c_{2}-f^{0}\left(v_{i}\right)\right)^{2}$. Therefore, the minimization of CV model on graphs is performed as an alternate minimization with respect to $\left(c_{1}, c_{2}\right)$ and with respect to $f$. Figure 1 shows the application of our CV model on
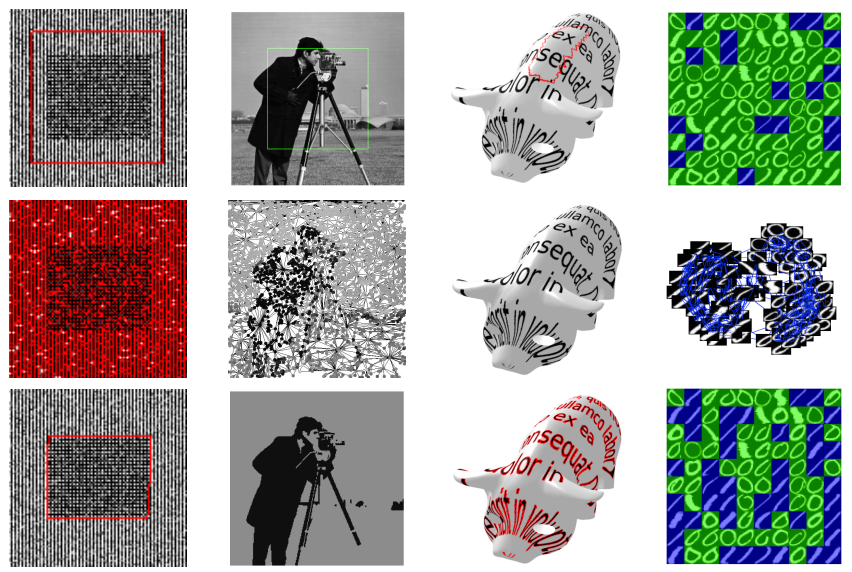

Fig. 1. Chan-Vese on various graphs (local and nonlocal, super-pixel, mesh, k-nearest neighbors) for image segmentation and data clustering.

many different graphs with $R_{w, 1}(f)$. First column presents a noisy textured image with an initial contour, the segmentation result on local (4-adjacency graph with Gaussian weights computed on pixel values) and nonlocal (4-adjacency graph coupled with a 4-Nearest Neighbor graph selected in a $9 \times 9$ window and Gaussian weights computed on $3 \times 3$ patches) graphs. The benefits of nonlocal patch-based configurations for image segmentation is evident for segmenting textured objects. Second column presents an image with an initial contour, the considered graph (a super-pixel graph obtained from an over-segmentation with Gaussian weights on region mean values), and the obtained partition of the super-pixel graph. This shows the interest of our formulation that can be used on a different representation of the image. Third column presents a mesh with an initial contour (on pig's back), the considered mesh (a triangular mesh with Gaussian weights on vertices grey values) with an image of text projected onto the surface, and the obtained partition of the mesh (all the text is extracted). Again, the algorithm remains the same, only graph changes. Our approach on meshes is therefore much more general than the recent framework proposed in [11]. Last column presents an example of high-dimensional data classification: an image database with an initial random partition, the considered graph (a 10 nearest neighbors graph weighted with Gaussian weights on $16 \times 16$ vectors associated to the image of each vertex). This shows how our CV models can be used in the space of images for classification; our CV model also corresponding a TV-regularized 2-means on graphs.

\section{CONCLUSION}

In this paper we proposed nonlocal PdEs on graphs for active contours models with applications to image segmentation and data clustering. Starting from nonlocal regularization functionals on graphs, we showed they verify the coarea formula on graphs and introduced nonlocal perimeters on graphs. These ingredients are then used for nonlocal active contours models as global minimizers for segmentation and clustering. Experimental results with the Chan-Vese models showed the benefits of the approach that enables to perform the binary partitioning of any graph.

\section{REFERENCES}

[1] D.K. Hammond, P. Vandergheynst, and R. Gribonval, "Wavelets on graphs via spectral graph theory," Appl. Comp. Harmonic Analysis, vol. 30, no. 2, pp. 129 - 150, 2011.

[2] L. Grady and C. Alvino, "The piecewise smooth Mumford-Shah functional on an arbitrary graph," IEEE Trans. on Image Proc., vol. 18, no. 11, pp. 2547-2561, 2009.

[3] A. Elmoataz, O. Lezoray, and S. Bougleux, "Nonlocal discrete regularization on weighted graphs: a framework for image and manifold processing," IEEE Trans. on Image Proc., vol. 17, no. 7, pp. 10471060, 2008.

[4] V.-T. Ta, A. Elmoataz, and O. Lézoray, "Nonlocal PDEs-based morphology on weighted graphs for image and data processing," IEEE Trans. on Image Proc., vol. 20, no. 6, pp. 1504-1516, June 2011.

[5] O. Lezoray, V.-T. Ta, and A. Elmoataz, "Partial differences as tools for filtering data on graphs," Patt. Rec. Letters, vol. 31, no. 14, pp. 2201-2213, 2010.

[6] L. Caffarelli, J.-M. Roquejoffre, and O. Savin, "Nonlocal minimal surfaces," Comm. on Pure and Applied Math., vol. 63, no. 9, pp. 1111$1144,2010$.

[7] X. Bresson and T.F. Chan, "Non-local unsupervised variational image segmentation models," CAM report 08-67, UCLA, 2008.

[8] X. Bresson, S. Esedoglu, P. Vandergheynst, J.-P. Thiran, and S. Osher, "Fast global minimization of the active contour/snake model," J. Math. Imaging and Vision, vol. 28, no. 2, pp. 151-167, 2007.

[9] N. Houhou, X. Bresson, A. Szlam, T. F. Chan, and J.-P. Thiran, "Semisupervised segmentation based on non-local continuous min-cut," in SSVM, 2009, vol. LNCS 5567, pp. 112-123.

[10] M. Nikolova, S. Esedoglu, and T.F. Chan, "Algorithms for finding global minimizers of image segmentation and denoising models," SIAM J. Appl.Math., vol. 66, no. 5, pp. 1632-1648, 2006.

[11] R. Lai and T.F. Chan, "A framework for intrinsic image processing on surfaces," Computer Vision and Image Understanding, 2011. 